\title{
Wichtiger als die „digitale Kluft“ : die „digitale Chance"
}

Hamadoun Ibrahim Touré and Michel Egger

\section{(2) OpenEdition}

\section{Journals}

Electronic version

URL: http://journals.openedition.org/sjep/543

DOI: $10.4000 /$ sjep.543

ISSN: 1663-9677

\section{Publisher}

Institut de hautes études internationales et du développement

\section{Printed version}

Date of publication: 1 novembre 2003

Number of pages: 119-128

ISSN: $1660-5926$

\section{Electronic reference}

Hamadoun Ibrahim Touré und Michel Egger, " Wichtiger als die "digitale Kluft" : die "digitale Chance“ », Schweizerisches Jahrbuch für Entwicklungspolitik [Online], 22-2 | 2003, Online erschienen am: 10 Juni 2010, abgerufen am 08 September 2020. URL : http://journals.openedition.org/sjep/543 ; DOI : https://doi.org/10.4000/sjep.543 


\title{
Wichtiger als die "digitale Kluft" : die "digitale Chance"
}

\author{
Hamadoun Ibrahim Touré
}

Im Gespräch mit Michel Egger

\begin{abstract}
D ie Internationale Fernmeldeunion (ITU) ist einer der wichtigsten Akteure beim institutionellen und infrastrukturbezogenen Ausbau der Telekommunikation in den Entwicklungsländern und in der Überwindung der digitalen Kluft. Hamadoun Ibrahim Touré, Direktor des Sektors für Telekommunikationsentwicklung der ITU (ITU-D), erläutert die Schwerpunkte seines Programms und nimmt Stellung zu einigen kontroversen Fragen.
\end{abstract}

Die Internationale Fernmeldeunion (ITU) ist die federführende Sonderorganisation der Vereinten Nationen bei der Organisation des Weltgipfels zur Informationsgesellschaft. Was bedeutet dies für den Sektor für Telekommunikationsentwicklung (ITU-D)?

Der Weltgipfel über die Informationsgesellschaft (WSIS) soll allen auf diesem Gebiet tätigen Akteuren als Katalysator dienen und es erlauben, laufende Projekte abzuschliessen und die gesetzten Ziele zu erreichen. Als Direktor des ITUD weiss ich selbst genau, welche Aufgaben ich in den kommenden vier Jahren bewältigen muss, denn diese wurden im März 2002 anlässlich der Weltkonferenz über die Telekommunikationsentwicklung in Istanbul definiert und im Oktober desselben Jahres durch die Konferenz der Regierungsbevollmächtigten in Marrakesch verabschiedet ${ }^{1}$. Dieses Programm muss ich umsetzen, Gipfel hin oder her, denn für diese Aufgabe wurde ich 1998 von der Konferenz der Regierungsbevollmächtigten in Minneapolis gewählt.

Die Frage lautet also: Wie kann ich den Gipfel und seine Dynamik nutzen, um meine Ziele so gut wie möglich zu erreichen, um mit meinen beschränkten Mitteln möglichst viel zu bewirken, und um Doppelspurigkeiten mit anderen Organisationen $\mathrm{zu}$ vermeiden? Auch heute noch ist dieser Bereich $\mathrm{zu}$ wenig koordiniert, so dass Mittel verschwendet werden. Der ITU-D hat beispielsweise im vergangenen Jahr in Thailand einen Kurs über den elektronischen Handel organisiert. Zwei Wochen später führte die Asia Pacific Telecommunity (APT) in Laos und in Kambodscha ähnliche Veranstaltungen durch. Dabei sind die Büros der APT gerade zwei Kilometer von unserer Niederlassung entfernt! Leider kommunizierten die beiden Ämter nicht miteinander und koordinierten ihre

1 Detaillierte Informationen über das ITU-Büro für Telekommunikationsentwicklung finden sich unter $<$ www.itu.int/ITU-D/>. 
Tätigkeiten nicht, obwohl ein bedeutender Teil der beitragzahlenden Mitglieder beiden Organisationen angehört. Natürlich haben wir Massnahmen ergriffen, um die Planung zu verbessern und um Synergien und Möglichkeiten zur Zusammenarbeit zu ermitteln. Dies ist nur eines von mehreren Beispielen.

Sowohl seitens der Regierungen und der Zivilgesellschaft als auch vom Privatsektor wurde Skepsis gegenüber dem Gipfel laut. Gewisse Länder fürchten einen Misserfolg, während andere insgeheim nicht unglücklich darüber wären, wenn der Gipfel zu keinen konkreten Ergebnissen führen würde ...

Aus meiner Sicht steht der Erfolg des Gipfels von vornherein fest. Was wirklich zählt, sind der Vorbereitungsprozess und die Energien und Ressourcen, die freigesetzt werden, um die digitale Kluft zu überwinden. Seit zwanzig Jahren ist sich die ITU dieses Problems bewusst. 1984 veröffentlichte die Maitland-Kommission einen Bericht von zentraler Bedeutung, der die weltweite Situation umfassend analysierte und feststellte, dass zwischen den Entwicklungsländern und den Industrienationen ein tiefer Graben verläuft. War damals die Rede vom „,fehlenden Bindeglied“ (missing link), spricht man heute von der „digitalen Kluft“. Das Phänomen wurde umbenannt, aber an den Tatsachen hat sich nichts geändert. Gestern ein Kettenglied, heute eine Kluft - und morgen mit Sicherheit wieder etwas anderes.

Damals setzte sich die ITU eine Frist von 20 Jahren, um den Graben zu überbrücken. Heute jedoch ist die Kluft tiefer als je zuvor. Hat die Politik der ITU versagt?

Die ITU hat vieles vollbracht, aber leider war es nicht genug. In diesem Sinn kann man von einem Misserfolg sprechen. Allerdings muss man auch den Hintergrund kennen. Zum einen stand die ITU allein da, zum anderen ist ihr Mandat begrenzt: Wir sind für die Infrastruktur zuständig, nicht für die Inhalte. Dafür sind andere Organisationen da. Wir können nicht alles selbst leisten. Wichtig ist dabei, sich nicht gegenseitig zu behindern und in der Zusammenarbeit die jeweiligen Kompetenzen zu achten. Will eine Organisation beispielsweise ein E-Learning-Projekt lancieren, so konzentriert sie sich am besten auf die inhaltliche Gestaltung, während wir sie bei den technischen Fragen im Zusammenhang mit der Errichtung eines Netzwerks unterstützen können. Aus dieser Sicht stellt der Gipfel eine einmalige Gelegenheit dar: Einerseits mobilisiert er die Kräfte unzähliger Akteure und ermöglicht Leistungen, welche die ITU nicht im Alleingang erbringen kann, andererseits verhindert er einen unkontrollierten Wettbewerb zwischen den einzelnen Organisationen. $\mathrm{Zu}$ oft versuchen einzelne Akteure, sich zu profilieren und das Verdienst für eine Initiative für sich zu beanspruchen. Dabei sollten wir doch alle Vorteile den armen Ländern zukommen lassen, anstatt uns darum zu streiten.

Welches sind die Tätigkeiten des ITU-D auf diesem Gebiet?

Unser Aktionsprogramm für die Jahre 2003 bis 2006 umfasst mehrere Schwerpunkte: 
$\square$ Die sektorielle Reform: Wie können die Länder bei der Errichtung eines soliden und ausreichend transparenten regulatorischen Umfelds unterstützt werden, welches die Entwicklung des Landes auf dem Gebiet der Informationsund Kommunikationstechnologien (IKT) gewährleistet, Investitionen anzieht und das Wachstum fördert?

$\sqcup$ Die ,E-Strategien“: Wie kann man den Regierungen helfen, eine Politik auszuarbeiten und umzusetzen, welche über Programme in den Bereichen $E$ Governance, E-Health, E-Learning, E-Business usw. sicherstellt, dass die sozialen und wirtschaftlichen Vorteile der IKT allen gesellschaftlichen Gruppen zugute kommen? Dabei geht es auch um die damit verbundenen technischen, rechtlichen und operativen Aspekte. Die Leitidee besteht darin, in gewissen Ländern Pilotprojekte zu lancieren.

$\square$ Der universale Zugang zu IKT, vor allem in ländlichen Gegenden. Wohlverstanden: Es geht hier um den universalen Zugang, nicht um die Universaldienstleistung. Denn zumindest zu Beginn ist es für uns wichtig, nicht einzelne Menschen in den Vordergrund zu rücken, sondern einen neuen Ansatz für die Entwicklung zu fördern, namentlich über die so genannten Telecenters, die den Gemeinschaften zur Verfügung stehen.

- Die Ausweitung und Vertiefung der Partnerschaften mit dem Privatsektor. Die ITU ist jene UNO-Sonderorganisation, die am meisten mit den Unternehmen zusammenarbeitet. Mehrere Firmen sind im ITU-D vertreten und arbeiten aktiv mit uns zusammen.

$\square$ Die vertiefte Analyse des Marktes und des Telekommunikationssektors. Damit wollen wir die Qualität unserer Informationen erhöhen, unsere Statistiken - die Bestseller der ITU, die weithin geschätzt werden - verbessern und unsere Prognoseinstrumente verfeinern. Wir hoffen, dass wir damit den Bedürfnissen der Wirtschaft noch besser gerecht werden können.

$\square$ Der Kapazitätsaufbau und die Entwicklung der personellen und technischen Ressourcen durch Bildungsprogramme, welche von den Universitäten, unseren eigenen Kompetenzzentren oder von den Internet-Akademien angeboten werden, die wir gemeinsam mit dem Unternehmen Cisco aufbauen. Damit wollen wir insbesondere die institutionellen und organisatorischen Kapazitäten der Länder stärken und gezielt jene Personen ansprechen, die Politiken ausarbeiten und umsetzen.

- Die Sonderprogramme zu Gunsten der am wenigsten entwickelten Länder $(L D C)$, welche es ermöglichen sollen, die erwähnten Zielsetzungen zu realisieren. Um die Effizienz zu steigern und der Mittelverschwendung vorzubeugen, haben wir beschlossen, uns während zwei Jahren auf zwölf Länder zu konzentrieren ${ }^{2}$. Wir versuchen, alle diese Länder unseren Partnern näher zu bringen, und hoffen, dass sie diese bei ihren Programmen ebenfalls berücksichtigen, denn dadurch werden Impuls und Wirkung maximiert. Zudem wurde eine besondere Initiative zu Gunsten einer Gruppe von LDC mit besonderen Bedürfnissen lanciert. Es handelt sich dabei um Länder, die einen Konflikt oder einen Bürgerkrieg hinter sich haben, wie zum Beispiel Afghanistan, Ruanda oder Sierra Leone.

2 Gemäss dem Arbeitsplan 2003 handelt es sich dabei um Bangladesch, Bhutan, Laos, Dschibuti, Burkina Faso, Malawi, Mali, Zentralafrikanische Republik, Republik Kongo, Somalia, Sambia und Haiti. 
Die letzte Konferenz der Regierungsbevollmächtigten in Marrakesch hat gezeigt, dass die ITU mit grossen finanziellen Schwierigkeiten zu kämpfen hat. Wie hoch ist Ihr Budget für dieses Programm?

Unser Budget beläuft sich auf 64 Millionen Franken pro Jahr. Dazu kommen 5 Millionen aus dem „Telecom Surplus Fund“. Das ist wenig, aber unsere Rolle besteht vornehmlich darin, günstige Rahmenbedingungen zu schaffen. In der Regel übernehmen wir selbst bis zu 25\% der Projektkosten, während die restlichen Investitionen über andere Partner getätigt werden. Die Mobilisierung und Bereitstellung dieser Mittel ist ein schwieriger, aber zentraler Aspekt unserer Tätigkeit. Um Gelder zu mobilisieren, ist ein entsprechendes Marketing erforderlich. Dieses verlangt jedoch gewisse Voraussetzungen. Aus diesem Grund habe ich strukturelle Reformen in die Wege geleitet, die namentlich die Transparenz verbessern sollen. Schliesslich will der Privatsektor wissen, wie sein Geld verwendet wird, und er verlangt zu Recht detaillierte Nachweise. Ich habe deshalb intern dafür gesorgt, dass wir diese Forderungen erfüllen können, was zuvor nicht immer der Fall war. Dass uns dies gelungen ist, beweist die Tatsache, dass wir heute mit Partnern wie Cisco zusammenarbeiten.

Gerade in Bezug auf die Partnerschaften mit Unternehmen wurde Kritik laut, wonach die ITU ihre Beziehungen mit dem weltweit tätigen amerikanischen Konzern Cisco zu stark privilegiert.

Ich bin der erste Direktor des ITU-D, der direkt aus der Privatwirtschaft kommt. Bereits in meiner vorherigen Stelle hatte ich Kontakt zur ITU, und ich kannte die Schwierigkeiten der Zusammenarbeit nur zu gut. Als wir unsere Zusammenarbeit mit dem Privatsektor begannen, war ich auf Kritik gefasst. Ich wusste, dass es alles andere als einfach sein würde, denn die Zusammenarbeit war damals im Entwicklungsbereich ein unbekanntes Konzept. Es galt, zahlreiche Hürden und Vorurteile zu überwinden, insbesondere die Befürchtung, die Unternehmen könnten den Namen der ITU für ihre eigenen Zwecke instrumentalisieren und missbrauchen.

Gewiss, unser Name hat einen Wert. Aber was nützt uns das, wenn wir nicht die Mittel haben, um ihn zu Gunsten jener zu nutzen, die es wirklich brauchen, nämlich für die Ärmsten dieser Welt? Bevor Cisco die Zusammenarbeit mit der ITU begann, erwirtschaftete das Unternehmen einen Jahresumsatz von 16 Milliarden Dollar. Glauben Sie wirklich, Cisco sei für ihren Erfolg auf uns angewiesen gewesen? Hingegen sind die Unternehmen aus ethischer Sicht der Menschheit etwas schuldig. Meine Aufgabe ist es, sie daran zu erinnern. Was kann daran falsch sein, wenn die ITU als Plattform dient, auf der die moralische und gesellschaftliche Verantwortung der Unternehmen konkrete Formen annimmt? Was spricht dagegen, mit den Unternehmen eine Partnerschaft zu schliessen und ihre Verpflichtung gegenüber der Menschheit zu „nutzen“? Dass die Unternehmen von dieser Zusammenarbeit profitieren, ist an sich nicht verwerflich. Was zählt, ist, dass diese Gewinne gerecht verteilt werden, dass Wachstum gefördert, Arbeitsplätze geschaffen und die Entwicklung vorangetrieben wird.

Was die privilegierten Beziehungen der ITU mit Cisco betrifft, so sind diese Vorwürfe absolut haltlos und ungerechtfertigt. Im Februar 1999, kurz nachdem ich 
meine Stelle angetreten hatte, schrieb ich mehr als 800 Unternehmen an und lud sie ein, uns bei bestimmten konkreten Vorhaben zu unterstützen. In anderen Worten: Sämtliche Unternehmen des Sektors wurden angesprochen. In meinem Schreiben hob ich zwei grundlegende Prinzipien hervor, die für jede Zusammenarbeit mit Unternehmen gelten, nämlich die Transparenz und die Nichtausschliesslichkeit. Unsere Tür steht damals wie heute allen Unternehmen offen, aber sie müssen von sich aus den Kontakt mit uns suchen. Cisco hat dies getan. Wenn wir heute eng mit Cisco zusammenarbeiten, dann nur deshalb, weil Cisco auf unseren Vorschlag eingegangen ist. Aber wie gesagt: Es ist genug Platz für alle da, denn ein einzelnes Unternehmen - auch ein Grosskonzern wie Cisco kann nicht den gesamten Bedarf abdecken. Wir setzen unsere Bemühungen zur Herstellung von Kontakten und zum Aufbau von Partnerschaften fort. Allerdings gehen wir dabei weitaus proaktiver vor, denn um Unternehmen zu mobilisieren, genügt es nicht, ihnen einen Brief zu schreiben. Wir selber haben zahlreiche Projekte vorzuschlagen, aber wir sind auch gegenüber anderen Vorschlägen offen, die sich gemeinsam konkretisieren und realisieren lassen.

Hat die ITU diese Nichtausschliesslichkeitsregel wirklich immer beachtet? Im Falle von Africa One beispielsweise, einem Paradebeispiel für einen „weissen Elefanten", hat die ITU mit AT\&T Submarine einen Vertrag über zwei Millionen Dollar abgeschlossen, ohne den Auftrag öffentlich auszuschreiben oder Alternativen zu prïfen ${ }^{3}$...

Dieser Fall hat sich noch vor meiner Zeit bei der ITU ereignet, und Sie werden verstehen, dass ich mich nicht dazu äussern kann. Es ist nicht meine Aufgabe, die Politik meines Vorgängers zu beurteilen. Alles, was ich dazu sagen kann, ist, dass die Dinge nicht so gelaufen sind, wie es hätte sein sollen...

Dann kann man also davon ausgehen, dass es sich bei diesem Projekt um eine Totgeburt handelt...

So ist es.

Bekanntlich ist Cisco ein Verfechter des IP (Internet-Protokolls), einer Technologie, die den Bedürfnissen der Entwicklungsländer nicht unbedingt entspricht ${ }^{4}$. Bietet die ITU mit der Errichtung von Cisco-Akademien in den afrikanischen Ländern dem amerikanischen Multi nicht eine kostengünstige Plattform für die Verwirklichung seiner Unternehmensziele? Eröffnet sie ihm damit nicht neue Absatzmärkte?

Der Grundsatz der Nichtausschliesslichkeit, ein zentrales Element unserer Politik, gilt nicht nur in Bezug auf die Unternehmen, sondern auch für Technologien und Ansätze. Das Internet-Protokoll ist eine von mehreren möglichen Lösungen, und es bleibt den einzelnen Ländern überlassen, ob sie diese übernehmen oder nicht. Im Gegensatz zur Weltbank oder zum Internationalen Währungsfonds zwingt die ITU keine Lösungsansätze auf. Überdies fehlen uns die finan- 
ziellen und politischen Mittel, um Druck auf einzelne Länder auszuüben. Unsere Argumente beziehen sich ausschliesslich auf die Technik.

Die von uns erarbeiteten Richtpläne oder Programme für sektorielle Reformen in den Entwicklungsländern bieten keine schlüsselfertigen Lösungen. Im Gegenteil: Wir bemühen uns, sämtliche verfügbaren Informationen und Möglichkeiten darzulegen und auf dieser Grundlage mit den politischen Verantwortlichen zusammenzuarbeiten, welche die Mittel und die Überzeugung haben, die gewählten Optionen erfolgreich umzusetzen.

Ich stamme selbst aus einem Entwicklungsland, und meine Aufgabe ist es, diese Länder zu unterstützen. Ich weiss, dass die Menschen in diesen Ländern nicht weniger intelligent und auch nicht weniger gebildet sind als ich selbst. Der einzige Unterschied liegt darin, dass sie keinen Zugang zu Information haben. Dabei ist Information die Grundlage für alles Weitere, unter einer Voraussetzung allerdings: Die Informationen müssen möglichst aktuell sein. Informationen sind wie Fische: Sie sind nur geniessbar, solange sie frisch sind. Deshalb müssen die Informationen den Entwicklungsländern zugänglich gemacht werden. Diese müssen möglichst aktuelle und möglichst vollständige Informationen erhalten, um Entscheidungen zu treffen und Lösungen zu wählen, die aus ihrer Sicht am günstigsten sind. Genau das ist unsere Aufgabe.

Der häufig kritisierte Nachteil dieses Vorgehens ist der Zeitaufwand. In der Tat benötigen wir bei diesem Vorgehen mehr Zeit, als wenn wir gebrauchsfertige Lösungen anbieten würden. Aber der grosse Vorteil ist, dass wir auf diese Weise dauerhafte Lösungen finden. Da sich die Entwicklungsländer selbst für eine Vorgehensweise entscheiden, die sie massgeblich mitgestaltet haben, werden sie nicht bei der ersten Schwierigkeit aufgeben oder uns um Hilfe rufen.

Hingegen zeigt die Erfahrung, dass aufgezwungene sektorielle Reformen nach einigen Jahren meistens ins Stocken geraten oder scheitern, weil niemand davon überzeugt ist. Problematisch ist, dass die Entwicklungsländer von anderen grossen internationalen Organisationen oft zu Politiken gezwungen werden, die ihren Interessen oder Überzeugungen widersprechen, und zwar aus dem einfachen Grund, weil ihnen das Wasser bis zum Hals steht und sie auf Darlehen oder andere Mittel dringend angewiesen sind. Sie wissen, wovon ich spreche: Ein armes Land, das auf das Geld der anderen angewiesen ist, hat nicht viel zu sagen, sondern muss die gestellten Bedingungen akzeptieren - Bedingungen übrigens, über die sich die anderen selbst gerne hinwegsetzen.

Um noch einmal auf die Cisco-Akademien zurückzukommen: Verschafft die ITU dem Unternehmen damit nicht Zugang zu einem Pool billiger künftiger Informatiker, wodurch der Braindrain noch verstärkt wird?

Der Braindrain hat eigentlich nichts mit den Cisco-Akademien zu tun. Es handelt sich um eine allgemeine Problematik, die so lange andauern wird, als es Entwicklungsunterschiede gibt. Heute benötigt der IKT-Sektor weltweit rund 300 '000 hoch qualifizierte Fachkräfte. Es gibt also einen Mangel, und daher ist es nicht verwunderlich, dass gut ausgebildete Leute aus den Entwicklungslän- 
dern von diesen in der Regel hoch bezahlten Jobs angezogen werden. Es ist ein natürliches Phänomen, das sich bestimmt nicht lösen lässt, indem Barrieren errichtet werden. Vielmehr gilt es, den Bildungsstand der Masse überall zu verbessern. Das Phänomen wird von selbst verschwinden, und zwar durch eine Nivellierung nach oben, nicht nach unten. Ich persönlich bin überzeugt, dass eine hoch qualifizierte Fachkraft, die das Land verlassen hat, besser ausgebildet zurückkehren wird.

Durch die strukturellen Reformen, die in der Regel auf Marktöffnung und Privatisierung beruhen, ist die ITU im Bereich der Telekommunikation zur Vorreiterin des Neoliberalismus geworden. Die Aussagen von Generalsekretär Yoshio Utsumi machen dies besonders deutlich. Die Liberalisierung ist aber nicht zwangsläufig ein Wundermittel für die LDC. Staatliche Monopole werden häufig durch privatwirtschaftliche Monopole abgelöst, die primär an den gewinnbringenden Marktbereichen interessiert sind...

Die Art, wie Sie diesen Sachverhalt darlegen, impliziert einen ideologischen oder dogmatischen Standpunkt der ITU. Dem ist aber nicht so. Wir verfolgen einen äusserst differenzierten Ansatz, der sich beispielsweise von jenem der Weltbank, welche die sofortige Privatisierung anstrebt, grundlegend unterscheidet. Ganz im Gegenteil: Wir waren praktisch die Einzigen, die darauf hingewiesen haben, dass es kein Patentrezept für alle Länder gibt. Privatisierungen sind nicht überall die geeignete Lösung, das hängt ganz von der jeweiligen Situation ab. Nicht alle Märkte besitzen eine Grösse, die für den Wettbewerb ausreichend oder geeignet ist. Wir anerkennen, dass es in den Ländern des Südens sehr erfolgreiche nationale Betreiber und Situationen gibt, in denen ein freier Markt nicht gerechtfertigt ist. Im Gegenzug haben verschiedene von uns durchgeführte Studien gezeigt, dass in gewissen Fällen die Privatisierung zu einer Verbesserung des Lebensstandards, zu einer Erhöhung der Abonnentenzahl, zu einer Steigerung der Dienstleistungsqualität und zu Preissenkungen geführt hat. Die ITU war beispielsweise an der Privatisierung der brasilianischen Anatel beteiligt. Das Ergebnis darf sich sehen lassen: Die anfänglichen Entlassungen wurden sehr rasch kompensiert, und der Personalbestand hat sich innerhalb von zwei Jahren verdoppelt. Sowohl für Inlands- wie für Auslandsgespräche sind die Tarife gesunken, und die Gewinne haben sich vervielfacht.

Die Grundregel lautet, dass man sich dem Rhythmus des jeweiligen Landes anpassen muss. In den Vereinigten Staaten und in der Europäischen Union wurden die Märkte mehr als hundert Jahre lang von öffentlichen Monopolen beherrscht, bevor die Privatisierung einsetzte. Wir wollen und können von den Entwicklungsländern nicht verlangen, sämtliche Zwischenschritte zu überspringen. Eine Entwicklung durchläuft unweigerlich gewisse Stadien. Die Privatisierung lässt sich nicht forcieren, sondern sie kann erst dann einsetzen und Früchte tragen, wenn alle Beteiligten - allen voran die Politiker und die obersten Verantwortlichen - daran glauben. Ist dies der Fall, werden sie sich nach Kräften dafür einsetzen, dass die Privatisierung zum Erfolg führt.

Ein schwerwiegendes Problem der Privatisierung - das in gewissen Entwicklungsländern besonders deutlich wurde - ist die Tatsache, dass Volksvermögen 
für ein „Butterbrot“ an Unternehmen aus dem Norden verschleudert wird, welche nur die Gewinn bringenden Sektoren instand setzen...

In der Tat sind in Ländern, die eine Privatisierung anstreben, die Netze häufig in einem schlechten Zustand. Sie müssten deshalb zuerst instand gesetzt werden, damit sie nicht unter ihrem Wert oder zu symbolischen Preisen verkauft werden müssen. Was privatisiert werden soll, muss in gutem Zustand sein. Häufig unterliegen jedoch gewisse staatliche Gesellschaften, die privatisiert werden sollen, den Strukturanpassungsprogrammen der Weltbank oder des IWF: Da es sich um staatliche Unternehmen handelt, dürfen sie keine Kredite aufnehmen, um zu investieren. Resultat: Die Unternehmen gehen zu Grunde, so dass man eine „Leiche“ verkaufen und schliesslich auch noch das Begräbnis bezahlen muss. Dem müsste aber nicht so sein, denn Telekommunikationsanbieter sind grundsätzlich rentable Unternehmen, die lediglich Investitionen und eine gute Unternehmensführung brauchen. Entgegen häufigen Behauptungen verfügen die Entwicklungsländer selbst über die Fachkräfte, die diese Unternehmen gut führen können. Dazu braucht es nicht unbedingt Ausländer.

Dies ist eines der Themen, das wir mit der Weltbank diskutieren. Man kann nicht eine gesamte Volkswirtschaft, alle Sektoren und sämtliche Unternehmen nach demselben Massstab beurteilen und ihnen dasselbe Rezept verschreiben, sondern es braucht einen verfeinerten, nach Branchen differenzierten Ansatz. Telekommunikationsanbieter dienen als Katalysator für die Entwicklung eines Landes: Man muss sie investieren und wachsen lassen, auch wenn es sich um staatliche Gesellschaften handelt.

Privatisierung hin oder her - ein minimaler „Service Public“ muss gewährleistet sein, und für den Wettbewerb müssen gewisse Spielregeln gelten, um Schäden und Missbräuche zu verhindern. Welche Rolle muss der Staat aus Ihrer Sicht spielen?

Die Rolle des Staates ist im Wesentlichen die eines Schiedsrichters und Katalysators. Damit widerspreche ich denjenigen, die behaupten, für den Staat sei in der Geschäftswelt kein Platz. Allerdings kann der Staat nicht gleichzeitig Schiedsrichter und Spieler sein, wie es der Fall war, als er gleichzeitig als Anbieter und als Regulator auftrat. Diese beiden Funktionen sind unvereinbar. Häufig sind die staatlichen Strukturen zu schwerfällig, als dass der Staat weiterhin als Spieler teilnehmen kann. Da er im Wettbewerb nicht mithalten kann, sollte er sich vorzugsweise auf seine Rolle als Schiedsrichter konzentrieren.

Ein offener Markt muss drei Voraussetzungen erfüllen: Erstens müssen Regeln festgelegt werden - das ist die Aufgabe des Staates. Zweitens müssen diese Regeln transparent und gerecht sein, so dass alle Wettbewerbsteilnehmer gleich behandelt werden, und drittens dürfen die Regeln während des Spiels nicht verändert werden, da sonst die Spieler das Feld verlassen.

Dies ist in der Tat der Idealfall, aber die Realität sieht häufig ganz anders aus. Es ist bekannt, dass in den „Führungsriegen“ gewisser Staaten Korruption herrscht, dass private und öffentliche Interessen miteinander verschmelzen oder aufeinander prallen... 
Zugegeben, dies ist ein Problem. Damit jedoch Korruption entsteht, muss ein korrumpierender Faktor, eine Versuchung, vorhanden sein. Aber - bei allem Respekt - diese Versuchung geht häufig von den Industrieländern aus. Allerdings glaube ich, dass sich die Dinge ändern. Zahlreiche Länder haben die erforderlichen Schritte eingeleitet, um dieser Situation zu entkommen, indem sie Fehlentwicklungen anerkannt, ihren Veränderungswillen bewiesen und adäquate Spielregeln ausgearbeitet haben. Heute verfügen 113 Länder - darunter zahlreiche Entwicklungsländer - über eine mehr oder weniger funktionierende Regulierungsbehörde. Das ist beachtlich. In manchen Entwicklungsländern haben sich die neuen Regeln und Strukturen günstig auf das Wachstum ausgewirkt, namentlich in Uganda, wo sich die Anschlussdichte innerhalb von vier Jahren verdreifacht hat. Die Zukunft wird weitere positive Entwicklungen bringen.

Sie sprechen häufig von Partnerschaften mit dem Privatsektor. Aber wie steht es mit der Zivilgesellschaft? Bis heute hat sich die ITU hartnäckig geweigert, sich Nichtregierungsorganisationen (NRO) zu öffnen. Dabei könnten diese doch gerade im Hinblick auf die Entwicklung eine wesentliche Rolle spielen. Steht diese Abschottung der ITU nicht im Widerspruch zur Philosophie des Weltgipfels über die Informationsgesellschaft, die einen wahrhaft tripartiten Prozess anstrebt, an dem Regierungen, Privatsektor und Zivilgesellschaft gleichermassen beteiligt sind?

Ich habe im vergangenen Januar in Tokio anlässlich der asiatisch-pazifischen Regionalkonferenz zur Vorbereitung des Gipfels wiederholt gesagt, dass wir den NRO offen gegenüber stehen und bereit sind, mit ihnen zusammenzuarbeiten. Wir sind aber der Ansicht, dass sie dazu nicht formell Mitglieder eines Sektors werden müssen. Wir wehren uns gegen die Politisierung der ITU. Wir engagieren uns in der Entwicklung, nicht in der Politik. Für Menschenrechtsdebatten sind andere Foren zuständig. Ich setze mich dafür ein, dass die Telekommunikation einen Beitrag zum Frieden und zur Wahrung der Menschenrechte leistet, aber ich bin nicht bereit, mich auf politische Diskussionen einzulassen.

Meine Aufgabe besteht darin, den Menschen die Mittel in die Hand zu geben, damit sie untereinander debattieren können, denn indem wir die Menschen miteinander kommunizieren lassen, fördern wir den Frieden. Dazu ist jedoch eine Infrastruktur erforderlich, wie zum Beispiel das Internet, Telefonanschlüsse, Verbindungen, Computer, Router, Energiequellen und so weiter. Sie bilden die Basis, auf der wir die Inhalte aufbauen können. Ohne diese sehr konkreten Strukturen und Instrumente gibt es keine Kommunikation und keine Übermittlung von Inhalten im erforderlichen Umfang und mit der benötigten Geschwindigkeit. Die ITU ist die einzige Organisation im Rahmen der Vereinten Nationen, die diese Infrastruktur bereitstellen kann.

In diesem Sinn sind wir natürlich zur Zusammenarbeit bereit, wenn eine NRO sich ernsthaft für die Entwicklung der Telekommunikationsinfrastruktur in den Ländern des Südens engagieren will. Nichts im Abkommen der ITU hindert uns daran, und zwar ungeachtet dessen, ob diese NRO Mitglied eines Sektors ist oder nicht. In Tokio habe ich folgenden Appell an die Zivilgesellschaft gerichtet: „Wir verlangen kein Geld von euch, sondern wir wünschen eure Mithilfe bei der Bewältigung unserer Aufgaben." 
Die ITU leidet unter mangelnder finanzieller Unterstützung ihrer Mitglieder. Offensichtlich unterstützen nur wenige Unternehmen den ITU-D...

Der ITU-D zählt 260 sektorielle Mitglieder. Das sind in der Tat weniger als in den beiden anderen Sektoren der ITU - Funkwesen und Standardisierung -, und auch die Grundbeiträge sind tiefer. Dabei gilt es zwei Aspekte zu bedenken: Erstens ist der ITU-D der jüngste Sektor der ITU - er wurde erst 1987 geschaffen und muss seinen Weg noch finden. Zweitens dient unsere Tätigkeit nicht direkt den Unternehmen, ganz im Gegensatz zu den anderen Sektoren wie beispielsweise bei der Standardisierung, wo die Unternehmen eigene kurz- und mittelfristige Interessen und Ziele verfolgen.

Aber die Zusammenarbeit macht Fortschritte. Zum Beispiel werden wir zusammen mit Swisscom und Sotelma (Société Nationale des Télécommunications du Mali) in Timbuktu ein Projekt „Schulen ans Internet“ lancieren ${ }^{5}$. Ich garantiere Ihnen, dass $80 \%$ des investierten Kapitals den eigentlichen Nutzern zugute kommt, und zwar als Ausrüstungsgüter, die im Land bleiben werden.

Dies ist ein weiteres Thema, das mir sehr am Herzen liegt: Die Entwicklungsländer haben genug von einer Entwicklungszusammenarbeit, die weniger ihrer Bevölkerung nützt als den beteiligten Unternehmen, genug von Projekten, die hauptsächlich der Imagepflege des grosszügigen Spenders dienen, während sich für die Betroffenen nichts ändert, und genug von der übertriebenen Budgethilfe, die grösstenteils in die Taschen der Sachverständigen und in die Villen der Berater fliesst.

Die ITU bemüht sich nach Kräften, dass ein möglichst hoher Prozentsatz der investierten Gelder effektiv in den betroffenen Ländern eingesetzt wird, und zwar nicht nur in Form von Ausrüstung, sondern über Bildungsanstrengungen auch in Form von Wissen.

\section{Wie beurteilen Sie die Vorbereitungsarbeiten zum Gipfel?}

Ein wesentlicher Aspekt ist, dass der Gipfel in zwei Phasen verläuft. Dies bietet die Möglichkeit, eine Zwischenbilanz zu ziehen. Im Dezember sollen in Genf wegweisende Ansprachen gehalten werden. Aber werden sie auch konkrete Ergebnisse zeitigen? Diese Frage werden wir im Herbst 2005 in Tunis beantworten können.

Ich persönlich beurteile die IKT als enorme Chance für die Entwicklung. Wenn es gelingt, die Frage der Telekommunikation zu entpolitisieren, dann kann sie eine grosse Chance bedeuten, und ein bedeutendes Potenzial für alle freisetzen: für die Unternehmen, für die Länder des Südens, aber auch für die Gesellschaften des Nordens. Was wirklich zählt, ist nicht die digitale Kluft, sondern die digitale Chance. 